\section{PROLONGED VIDEO-EEG NECESSARY TO IDENTIFY NON-CONVULSIVE STATUS EPILEPTICUS IN INFANTS ADMITTED TO ICUS}

\section{T. Gilboa}

\section{Shaare Zedek Med Ctr, Jerusalem, Israel}

Electrographic seizures are electrical events that can be detected by EEG but do not have clear clinical correlation. Despite the fact that these are found in over $40 \%$ of unconscious patients in intensive care units, many physicians are not aware of it. In the recent years it became clear that children and infants also suffer from it. Moreover, non-convulsive status epilepticus as a cause of decreased mental status is prevalent in children more than in adults. We present 2 cases of infants who were admitted to the NICU and PICU, were unconcsious and their routine EEG did not show epileptic activity. Only prolonged Video EEG demonstrated non-convulsive status epilepticus and the treatment was changed accordingly.

Electrographic seizures in unconscious infants are prevalent. Though the long term consequences of these events are yet to be determined, in adults the delay in diagnosis and treatment is associated with poor prognosis. The closest entity in pediatrics that maybe used as a model is electrical status epilepticus in sleep. This is a pediatric disorder associated with cognitive and behavioral regression and carries a poor prognosis. It is unclear weather the seizures themselves are the offending cause but early diagnosis and treatment increase the probability of recovery.

A prolonged Video-EEG monitoring is a safe, available and reliable test to diagnose this. The literature and these two patients demonstrate the benefit of early use of the test and the immediate implications on the treatment.

\section{5}

\section{NEURO DEGENERATION IN CHILDREN: DEVELOPING COUNTRY PERSPECTIVES}

T. Sultan ${ }^{1}$, A.W. Rathore ${ }^{2}$

${ }^{1}$ Paediatric Neurology, Institute of Child Health Lahore Pakistan, ${ }^{2}$ Paediatric Medecine, Children Hospital Lahore, Lahore, Pakistan

Back ground and aims: To find out the spectrum of neurodegenerative disorders of childhood, its diagnosis and role of paediatician in the management. It isa descriptive study conducted at Department of Neurology Children's Hospital, Lahore from June 1, 2005 to May 31, 2009.

Methods: A total of 2273 patients were admitted in the Neurology department with the diagnosis of DBD.

Results: Male to female ratio was 1.4:1. Age range was one to fifteen years. Metachromatic leukodystrophy was the predominant type (21\%) followed by adrenoleukodystrophy (16\%), SSPE $(12 \%)$, Wilson Disease $10 \%$, Alexander disease $3 \%$, Hellervordenspatz disease $2 \%$ and canavans leukodystrophy in $1.5 \%$.

Conclusion: Degenerative brain diseases are not uncommon entity in paediatric population. Commonest presentation is regression of mile stones though it may be variable. Physicians must look into it when dealing with children having regression of milestones to diagnose them earlier. As in majority of patients there is yet no curative therapy available, so role of palliative care is very important. Collaboration should be established between the regional institutes to promote further research in future.

\section{6}

\section{CONGENITAL MYOTONIC DYSTROPHY: SMOOTH MUSCLE INVOLVEMENT OF THE URINARY TRACT IN A 2 YEAR OLD CHILD}

\author{
V. Mundada, T. Hildebrandt \\ Paediatrics, Princess of Wales Hospital, \\ Bridgend, UK
}

We report a child with Congenital Myotonic Dystrophy (CDM1) who presented at the age of 28 months with marked smooth muscle involvement of the urinary tract. Congenital Myotonic Dystrophy is a multi-system disorder affecting skeletal, smooth and cardiac muscles. Smooth muscle involvement predominantly affects the gastrointestinal tract. Involvement of the urogenital smooth muscles is extremely rare and has so far only reported in adult cases. There is little published on the effects of myotonia on the detrusor muscle.

The index case was a female infant who was the first child of non-consanguineous Caucasian parents. The antenatal ultrasound scan at 31 weeks showed evidence of polyhydromnia and moderate right renal pelvic dilatation. She was born at 33 weeks gestation by spontaneous vaginal delivery. Her mother had 